\title{
Effect of quantum noise on Coulomb blockade in normal tunnel junctions at high voltages
}

\author{
J.S. Penttilä ${ }^{1}$, Ü. Parts ${ }^{1}$, P.J. Hakonen ${ }^{1}$, M.A. Paalanen ${ }^{1}$, and E.B. Sonin ${ }^{2}$ \\ ${ }^{1}$ Low Temperature Laboratory, Helsinki University of Technology, FIN-02015 HUT, Finland \\ ${ }^{2}$ The Racah Institute of Physics, The Hebrew University of Jerusalem, Jerusalem 91904, Israel
}

(June 9, 2021)

\begin{abstract}
We have investigated asymptotic behavior of normal tunnel junctions at voltages where even the best ohmic environments start to look like RC transmission lines. In the experiments, this is manifested by an exceedingly slow approach to the linear behavior above the Coulomb gap. As expected on the basis of the quantum theory taking into account interaction with the environmental modes, better fits are obtained using $1 / \sqrt{V}$ - than $1 / V$-dependence for the asymptote. These results agree with the horizon picture if the frequency-dependent phase velocity is employed instead of the speed of light in order to determine the extent of the surroundings seen by the junction.
\end{abstract}

PACS numbers: 74.50.+r, 73.23.-b, 73.23.Hk

\section{INTRODUCTION}

Coulomb blockade in a single normal tunnel junction is strongly affected by the environment if the real part of impedance is less than the quantum resistance $R_{K}=h / e^{2} \approx 26 \mathrm{k} \Omega$. However, a quite general sum rule requires that, at high voltages, the $I V$ curve is of the form $V=I R+e / 2 C$ where $C$ is the geometric capacitance of the tunnel junction [1]. The environment strongly influences the way how this asymptote is approached. Experiments on typical samples with low resistance leads exhibit asymptotic $1 / V$ tails which can be explained well using either the quantum theory of environment [1], or the horizon model [2,3].

In this paper we analyze our experimental results on $I V$ curves for a variety of single small isolated tunnel junctions, shunted and unshunted, with different values of capacitance $C$ and tunneling resistance $R_{T}$. Our attention is concentrated especially on the high-voltage part of the $I V$ curves obtained for samples with highly resistive Cr leads. At high frequencies which are most important for the high-voltage asymptotics such leads behave as lossy $R C$ lines. Experimental results are discussed and interpreted in terms of the quantum theory of environment which predicts that the high-voltage asymptote is approached as $1 / \sqrt{V}$, i.e. much slower than $1 / V$ tails revealed for a purely real impedance. Indeed, we find asymptotic $1 / \sqrt{V}$ tails experimentally in the range of voltages $1-10 \mathrm{mV}$. An important feature of our experimental conditions is that the junction resistance for some samples is not large compared with $R_{K}$ and the strong tunneling corrections have to be taken into account.

We start this paper with a theoretical overview (Sec. II) which concentrates on predictions of the quantum theory of electromagnetic environment [1] for the highvoltage asymptotics of $I V$ curves. This theory accounts for environmental effects by a phase dependent factor in the tunnel Hamiltonian which describes the tunneling rate of electrons through the junction. Because of the Johnson-Nyquist noise in the electric circuit, the phase fluctuates and this affects the tunneling rate. Due to exchange of energy between the electron and environmental modes, the delta-function $\delta(E)$ in energy must be replaced by a broader distribution function $P(E)$ in the expression for the tunneling rate. Therefore, this is called $P(E)$ theory [3]. We prefer the term phase-correlation theory, introduced in Ref. 4, emphasizing the important role of phase fluctuations and phase memory in the theory. An alternative voltage-fluctuation theory suggested in Ref. [1], is also discussed. The latter, in contrast to the phase-correlation theory, predicts an exponential tail for the high-voltage asymptotics.

The effect of environment is less pronounced in the high-voltage than in the low-voltage part of the $I V$ curve, and therefore it is more difficult for scrutiny. But we show in the theoretical overview that the high-voltage asymptotics is governed by only the quantum part of the Johnson-Nyquist noise, and therefore is quite important for a reliable comparison with the quantum theory. The high-voltage asymptotic is also convenient for studying strong tunneling corrections which are important at our experimental conditions. In Sec. II we argue that a proper account of strong tunneling corrections is to include the junction resistance as a lumped element in an effective electric circuit used for calculation of the Johnson-Nyquist noise. This view is proven by comparing this approach with a more elaborate theoretical analysis [5, 5 .

The experiment and its comparison with the theoretical predictions is described in Sec. III. We fit the experimental high-voltage tails by a combination of $1 / V$ and $1 / \sqrt{V}$ tails with their amplitudes as fitting parameters, and compare the outcome with the values calculated for our effective electric circuit. Note that experimentally it is rather hard to distinguish between different power-law dependences on voltage unless separately determined pa- 
rameters are employed in restricting the fitted formulas. But there is a clear difference between the power-law and exponential tails, and our fitting is in favor of the powerlaw tails predicted by the phase-correlation theory. The paper is concluded by discussion (Sec. IV).

\section{THE $I V$ CURVE ASYMPTOTICS}

\section{A. The phase-correlation theory}

The $I V$ curve is given by

$$
I=e\left[\Gamma^{+}(V)-\Gamma^{-}(V)\right]
$$

where the forward tunneling probability reads [1]

$$
\begin{gathered}
\Gamma^{+}(V)=\frac{1}{e^{2} R_{T}} \int_{-\infty}^{\infty} d E \int_{-\infty}^{\infty} d E^{\prime} f(E)\left[1-f\left(E^{\prime}\right)\right] \\
\times \int_{-\infty}^{\infty} \frac{d t}{2 \pi \hbar} \exp \left[\frac{i t}{\hbar}\left(E-E^{\prime}+e V\right)\right]\left\langle e^{i \varphi(t)} e^{-i \varphi(0)}\right\rangle
\end{gathered}
$$

and the backward tunneling rate $\Gamma^{-}(V)=\Gamma^{+}(-V)$. Here $f(E)$ is the Fermi distribution, and $\varphi(t)=$ $\frac{e}{\hbar} \int_{-\infty}^{t} d t^{*} \delta V\left(t^{*}\right)$, is the fluctuation of the phase difference due to fluctuating voltage $\delta V$ across the junction, which is treated as a quantum-mechanical operator. The averaging $\langle\ldots\rangle$ is performed over possible states of quantum environment, i.e. an ensemble of modes in an electric circuit which the junction is embedded in. If the phase does not fluctuate, the integral $\int d t$ in Eq. (2) yields the energy delta-function $\delta\left(E-E^{\prime}+e V\right)$, and Eq. (2) reduces to the usual expression for an ohmic tunnel junction. But taking into account the phase fluctuations, Eq. (2) yields after some algebra (see Eq. (56) in Ref. [1]):

$$
\Gamma^{+}(V)=\frac{1}{e^{2} R_{T}} \int_{-\infty}^{\infty} d E \frac{E}{1-\exp \left(-\frac{E}{k_{B} T}\right)} P(e V-E)
$$

Here

$$
P(E)=\frac{1}{2 \pi \hbar} \int_{-\infty}^{\infty} d t \exp \left[J(t)+\frac{i E t}{\hbar}\right],
$$

and

$$
\begin{aligned}
J(t) & =\langle[\varphi(t)-\varphi(0)] \varphi(0)\rangle \\
& =2 \int_{-\infty}^{\infty} \frac{d \omega}{\omega} \frac{\operatorname{Re} Z(\omega)}{R_{K}} \frac{e^{-i \omega t}-1}{1-e^{-\beta \hbar \omega}}
\end{aligned}
$$

is the phase-correlation function where $\beta=1 / k_{B} T$. Since $\operatorname{Re} Z(\omega)$ is an even function of $\omega$, the imaginary part of $J(t)$ does not depend on temperature:

$$
\operatorname{Im} J(t)=-\int_{-\infty}^{\infty} \frac{d \omega}{\omega} \frac{\operatorname{Re} Z(\omega)}{R_{K}} \sin (\omega t) .
$$

Using the time-domain formulation [7] 8 ] one can rewrite Eq. (3):

$$
\Gamma^{+}(V)=\frac{1}{2 \pi \hbar e^{2} R_{T}} \int_{-\infty}^{\infty} d t \gamma(t) \exp \left[J(t)+\frac{i e V t}{\hbar}\right],
$$

where

$$
\begin{aligned}
\gamma(t) & =\int_{-\infty}^{\infty} d E \frac{E}{1-\exp \left(-\frac{E}{k_{B} T}\right)} \exp \left(-\frac{i E t}{\hbar}\right) \\
& =i \pi \hbar^{2} \frac{d}{d t} \delta(t)-\frac{\pi^{2}}{\beta^{2}} \frac{1}{\sinh ^{2}(\pi t / \hbar \beta)} .
\end{aligned}
$$

Then using the sum rules $J(0)=0$ and $i J^{\prime}(0)=e^{2} / 2 C \hbar$ [1], the current becomes

$$
\begin{array}{r}
I=\frac{1}{R_{T}}\left[V-\frac{e}{2 C}+\frac{\pi}{e \hbar \beta^{2}}\right. \\
\left.\times \int_{-\infty}^{\infty} \frac{d t}{\sinh ^{2}(\pi t / \hbar \beta)} \operatorname{Im}\left\{e^{J(t)}\right\} \sin \frac{e V t}{\hbar}\right] .
\end{array}
$$

The effect of the environment is given by the last term. The second derivative of the current at high $V \gg \pi / e \beta=$ $\pi k_{B} T / e$ becomes

$$
\frac{d^{2} I}{d V^{2}}=-\frac{e}{\pi \hbar R_{T}} \int_{-\infty}^{\infty} d t \operatorname{Im}\left\{e^{J(t)}\right\} \sin \frac{e V t}{\hbar}
$$

The asymptotic behavior at $V \rightarrow \infty$ is determined by the short-time expansion of the correlation function $J(t)$ where it is small. Using Eq. (6) we obtain

$$
\begin{aligned}
\frac{d^{2} I}{d V^{2}} & =-\frac{e}{\pi \hbar R_{T}} \int_{-\infty}^{\infty} d t \operatorname{Im}\{J(t)\} \sin \frac{e V t}{\hbar} \\
& =\frac{e}{\pi \hbar R_{T}} \int_{-\infty}^{\infty} d t \int_{-\infty}^{\infty} \frac{d \omega}{\omega} \frac{\operatorname{Re} Z(\omega)}{R_{K}} \sin (\omega t) \sin \frac{e V t}{\hbar} \\
& =\frac{2}{R_{T} R_{K}} \frac{\operatorname{Re} Z(e V / \hbar)}{V} .
\end{aligned}
$$

The total impedance of the circuit may be presented as $Z^{-1}(\omega)=Y_{0}(\omega)-i \omega C$, where the admittance $Y_{0}(\omega)$ refers to the whole circuit except for the capacitive channel of the tunnel junction. At high frequencies (voltages) one has:

$$
\frac{d^{2} I}{d V^{2}} \approx \frac{e^{2} R_{K}}{2 \pi^{2} C^{2} R_{T}} \frac{\operatorname{Re} Y_{0}(e V / \hbar)}{V^{3}}
$$

Now integrating twice from $V$ to $\infty$ we obtain the $I V$ curve

$$
I=\frac{1}{R_{T}}\left[V-\frac{e}{2 C}+V_{t}(V)\right]
$$

where the "tail" voltage is

$$
V_{t}=\frac{e^{2} R_{K}}{2 \pi^{2} C^{2}} \int_{V}^{\infty} d V_{1} \int_{V_{1}}^{\infty} d V_{2} \frac{\operatorname{Re} Y_{0}\left(e V_{2} / \hbar\right)}{V_{2}^{3}}
$$


Alternatively, the effect of the tail can be presented as a voltage dependent correction to the junction capacitance:

$$
I=\frac{1}{R_{T}}\left(V-\frac{e}{2 \tilde{C}(V)}\right)
$$

where the voltage dependent capacitance $\tilde{C}$ is

$$
\tilde{C}(V)=C\left(1+\frac{C}{e} V_{t}(V)\right) .
$$

In the ohmic case, $Y_{0}=R^{-1}$ and

$$
V_{t}=\frac{R_{K}}{R}\left(\frac{e}{2 \pi C}\right)^{2} \frac{1}{V} .
$$

Then Eq. (13) agrees with Eq. (115) of Ref. [1].

Thus the $I V$ curve at high voltages scans the highfrequency impedance and does not depend on temperature. This means that only the quantum noise of environment affects the high-voltage behavior, and this is manifested by proportionality of high-voltage tails to the quantum resistance $R_{K}$. But one must remember that the derived asymptotic behavior starts at voltages $V$ higher than $\pi k_{B} T / e$. This could make the observation of asymptotic tails impossible at very high temperatures because of their very small contribution which could be masked by nonlinear corrections to the junction conductance at very high voltages.

\section{B. Alternative theory of the environment effect: voltage-fluctuation theory}

There is another approach to take into account environmental modes on the tunneling rate. One uses the expression for the tunneling probability obtained for a static voltage at the junction assuming that this voltage is a random quantity described by the Gaussian distribution arising from the Johnson-Nyquist noise. Let us call it the voltage-fluctuation theory. Initiated by

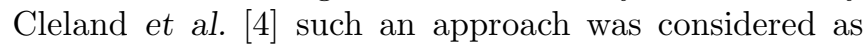
a heuristic model, in contrast to "the more accurate" phase-correlation model. We argue that the two approaches are not at all equivalent when compared with each other. They are essentially different in the physical picture of the phenomenon. The phase-correlation theory assumes that phase fluctuations affect the tunneling probability via a phase-dependent factor in the tunneling Hamiltonian. This phenomenon represents an example of a "phase-memory effect". In contrast, the voltagefluctuation theory assumes that the tunneling probability depends only on the voltage at the present moment, without any memory effect. But the voltage fluctuates due to the Johnson-Nyquist noise in the circuit and this affects the current through the junction.
In spite of completely different starting assumptions, the results of the voltage-fluctuation and the phasecorrelation theories agree on certain aspects. In particular, both the theories demonstrate that quantum fluctuations of the environment become important when the circuit resistance $R$ becomes of the order of the quantum resistance $R_{K}$. However, there is an essential difference in predictions for the following effect: At low voltages when the Coulomb effects are the most pronounced, the voltage-fluctuation theory predicts an ohmic behavior for zero-bias anomaly, but with an exponentially large resistance value compared with the nominal tunneling resistance $R_{T}$. In contrast, the phase-correlation theory [1] gives a nonanalytic $I V$ curve with a non-linear power law. At high voltages the voltage-fluctuation theory predicts an exponentially decreasing quantum-fluctuation correction to the $I V$ curve, against a power-law decrease in the phase-correlation theory as derived above.

These two theories were compared in experiments by Farhangfar et al. [9] at low voltages, and this comparison was in favor of the phase-correlation theory. The present paper addresses the $I V$ curve at high-voltages and we shall discuss predictions of the voltage-fluctuation theory for the asymptotics of the $I V$ curve. Forward and backward tunneling probabilities at zero temperature are

$$
\Gamma^{ \pm}(V)=\frac{1}{e R} \int_{\frac{e}{2 C} \mp V}^{\infty}\left( \pm V+\delta V-\frac{e}{2 C}\right) p(\delta V) d \delta V .
$$

Here $p(\delta V)$ is the voltage fluctuation probability distribution which we assume to be Gaussian:

$$
p(\delta V)=\frac{1}{\sqrt{2 \pi} \Delta V} \exp \left(-\frac{\delta V^{2}}{2 \Delta V^{2}}\right),
$$

and $\Delta V=\sqrt{\left\langle\delta V^{2}\right\rangle}$ is the standard deviation of this distribution. Then after integration in the limit $V \gg$ $e / 2 C, \Delta V\left(\Gamma^{-}\right.$is insignificant in this limit):

$$
\Gamma^{+}=\frac{1}{e R}\left\{V-\frac{e}{2 C}+\frac{\Delta V^{3}}{\sqrt{2 \pi} V^{2}} \exp \left[-\frac{V^{2}}{2 \Delta V^{2}}\right]\right\} .
$$

This yields the exponential asymptotic voltage tail

$$
V_{t}=\frac{\Delta V^{3}}{\sqrt{2 \pi} V^{2}} \exp \left(-\frac{V^{2}}{2 \Delta V^{2}}\right) .
$$

Similar to phase fluctuations, voltage fluctuations are determined by the Johnson-Nyquist noise in the circuit and

$$
\Delta V^{2}=\frac{\hbar^{2}}{e^{2}}\left\langle\dot{\varphi}(0)^{2}\right\rangle=\frac{\hbar}{\pi} \int_{-\infty}^{\infty} \omega d \omega \frac{\operatorname{Re} Z(\omega)}{1-e^{-\beta \hbar \omega}} .
$$

Thus, in contrast to the phase-correlation theory, the high-voltage asymptote is affected not by a single noise 
mode with frequency $\omega=e V / \hbar$, but by the whole noise spectrum. As a result, the voltage tail must decrease exponentially, which is not confirmed in our experiments (see Sec. [III). In the rest of this section we restrict ourselves with discussion of the phase-correlation theory.

\section{Strong tunneling effects}

Originally, the phase-correlation theory was developed for the weak tunneling regime, where the junction resistance $R_{T}$ is large $\left(\gg R_{K}\right)$ and its contribution to the equilibrium noise in the circuit is negligible. In our experiment $R_{T} \sim R_{K}$ and we must include also the strong tunneling effects. Usually tunneling is defined as strong when the junction resistance becomes of the order or smaller than $R_{K}$. But in fact one must take into account strong tunneling effects even if $R_{T}$ becomes comparable with the circuit resistance. Let us consider the case when $R_{T}$ is the smallest of all parallel resistors in the circuit. Then $Y_{0} \approx 1 / R_{T}$ and

$$
I=\frac{1}{R_{T}}\left[V-\frac{e}{2 C}\left(1-\frac{R_{K}}{R_{T}} \frac{e}{2 \pi^{2} C} \frac{1}{V}\right)\right] .
$$

This asymptotic expression for the $I V$ curve looks like an expansion in the parameter $\alpha e / C V$ where $\alpha=R_{K} / R_{T}$ characterizes dissipation. The same parameter determined strong tunneling effects analyzed by Odintsov [5] (see also the expression for the $I V$ curve and discussion in Sec. 5.3.4 of Ref. [10]). Expanding the expression for the $I V$ curve of Odintsov in $\alpha e / C V$, the first terms of the expansion exactly reproduce Eq. (23) derived as an asymptotic expression for high voltages. The results by Odintsov [5] have also been confirmed by a more general analysis of strong tunneling by Golubev and Zaikin [6] using path-integral technique.

Thus, at least at high voltages, strong tunneling effects can be taken into account simply by including the tunnel resistance $R_{T}$ into the effective electric circuit. This means that $R_{T}$ contributes to the equilibrium JohnsonNyquist noise on equal terms with other dissipative elements of the circuit. The same approach was used by Joyez et al. [8] in the analysis of the low-voltage part of the $I V$ curve, but instead of using $R_{T}$ as an element of the electric circuit to account for the noise from the junction, they used the differential resistance at zero-voltage bias, which may be much larger than $R_{T}$.

\section{Circuit elements as transmission lines}

At high frequencies resistors in the circuit cease to be lumped elements and should be considered as transmission lines with distributed resistance, capacitance, and inductance. The admittance of a double transmission line shown in Fig. 1 (drawn without inductive elements) is

$$
Y_{L}(\omega)=\frac{1}{2} \sqrt{\frac{i \omega C_{R}}{R-i \omega L}} \cot \sqrt{i \omega C_{R}(R-i \omega L)}
$$

where $R, L$, and $C_{R}$ are the total resistance, inductance, and capacitance of an individual transmission line. At low frequency $\left(\omega \ll 1 / R C_{R}, R / L\right)$, the admittance becomes purely ohmic, i.e., it behaves as a lumped ohmic resistor: $Y_{L} \approx 1 / 2 R$.

For a low-resistance transmission line with $R \ll$ $\sqrt{L / C_{R}}$, the resistance may be neglected, but in the high-frequency limit the double transmission line behaves, nevertheless, as an ohmic resistor with a real impedance $2 \sqrt{L / C_{R}}$ (the energy is lost via radiation along an infinite transmission line). Then the voltage tail is $\propto 1 / V$ as for a lumped resistor:

$$
V_{t}=\frac{R_{K}}{2} \sqrt{\frac{C_{R}}{L}}\left(\frac{e}{2 \pi C}\right)^{2} \frac{1}{V} .
$$

But for the high-resistance line with $R \gg \sqrt{L / C_{R}}$ one may neglect inductance, and the real part of the admittance in the high-frequency limit becomes

$$
\operatorname{Re} Y_{L}(\omega) \approx \frac{1}{2} \operatorname{Re} \sqrt{\frac{-i \omega C_{R}}{R}}
$$

Then the voltage tail decreases slower, as $1 / \sqrt{V}$ :

$$
\begin{aligned}
V_{t} & =\frac{a_{1 / 2}}{V^{1 / 2}} \\
a_{1 / 2} & =\frac{1}{3 \pi^{3 / 2}} \sqrt{\frac{R_{K} C_{R}}{R C}}\left(\frac{e}{C}\right)^{3 / 2} .
\end{aligned}
$$

But irrespective of the magnitude of the dissipative component, at very high frequency $\omega \gg R / L$ (high voltage $V \gg(\hbar / e) R / L))$ the (double) transmission line becomes again ohmic with the real impedance $Z_{L} \approx 2 \sqrt{L / C_{R}}$ which is much smaller than $R$ if the line is long (since $R$ is proportional to the line length, but $L / C_{R}$ is not). However, if $R$ is large enough, this happens for voltages too high to be relevant in the experiments. Altogether, both high-voltage tails, Eqs. (25) and (27), become valid when the "uncertainty" time $\tau_{V}=\hbar / \mathrm{eV}$ introduced by Nazarov [11] becomes less than the relaxation time $R C_{R}$ of the circuit.

\section{E. Stray capacitance and the horizon model}

One way to describe the high-frequency effect of environment is to use the so-called "horizon model" [3, 12]. It represents the effect of the environment as due to stray capacitance of leads described by transmission lines. The relevant stray capacitance originates from the length of 
the transmission line over which an electromagnetic signal from the junction can travel during the uncertainty time $\tau_{V}=\hbar / e V$. This length is called the "horizon" length $v_{p h} \tau_{V}$. Here $v_{p h}$ is the velocity of the signal propagation. Indeed, the effective voltage dependent capacitance incorporating the effect of the voltage tail (see Eqs. (15,16)) can be presented as

$$
\tilde{C}=C+\frac{C^{2}}{e} V_{t}(V)=C+c_{R} v_{p h} \tau_{V},
$$

where $c_{R}=C_{R} / \mathcal{L}$ is the capacitance per unit length and $\mathcal{L}$ is the length of the transmission line. In the past [3, 32 ] the horizon model was used for lossless (low-resistance) transmission lines when

$$
\tilde{C}=C+\frac{1}{2 \pi^{2}} \sqrt{\frac{C_{R}}{L}} \frac{h}{e V}
$$

and $v_{p h}=1 / \sqrt{c_{R} l}$ is of the order of the light velocity (here $l=L / \mathcal{L}$ is the inductance per unit length). But the model works also for lossy (high-resistance) lines when

$$
\begin{aligned}
\tilde{C}=C+\frac{4}{3 \pi^{3 / 2}} \sqrt{\frac{R_{K} C_{R}}{R C}}(e C)^{1 / 2} \frac{1}{V^{1 / 2}} \\
=C+\frac{4}{3 \pi^{3 / 2}} \sqrt{\frac{h C_{R}}{R e V}} .
\end{aligned}
$$

Comparing it with Eq. (28) one sees that $v_{p h} \sim$ $\sqrt{\omega / c_{R} r} \sim \sqrt{e V / \hbar c_{R} r}$, and again on the order of the phase velocity along the transmission line (here $r=R / \mathcal{L})$, but for the lossy line this velocity is frequency(voltage)-dependent and much less than the speed of light. Thus, the horizon picture presents a good qualitative picture of the effect of environment on the high-voltage asymptotics.

Originally the horizon model was introduced by Büttiker and Landauer [13], who assumed the characteristic time to be the traversal time of tunneling which is extremely small (about $10^{-15} \mathrm{sec}$ ). In fact, Büttiker and Landauer 13 considered the process of tunneling itself which indeed can be affected by the circuit only on such short time scales. In the phase-correlation theory 1] the circuit noise influences the phase factor in the tunneling Hamiltonian (i.e. the phases of the quantum states on both sides of the junction), but not the tunneling amplitude itself which is characterized by constant junction conductance $1 / R_{T}$. This influence is possible over a distance which is the horizon length determined by the uncertainty time $\hbar / \mathrm{eV}$.

\section{EXPERIMENT}

\section{A. Description of samples}

Our sample consists of a aluminum tunnel junction (area $150 * 150 \mathrm{~nm}^{2}$ ), shunted or unshunted, connected to four measurement leads via thin film $\mathrm{Cr}$ resistors $(25$ $\mu \mathrm{m}$ long) that are located within $3 \mu \mathrm{m}$ from the junction (see inset of Fig. 2). The circuits were fabricated using electron beam lithography and triple-angle evaporation on top of oxidized silicon substrate $\left(\mathrm{SiO}_{2}\right.$ thickness $\sim$ $100 \mathrm{~nm}$ ). The tunnel barriers were formed by oxidizing the bottom aluminum electrode in $\mathrm{O}_{2}$ at 0.1 mbar for 5 minutes. The $\mathrm{Cr}$ resistors and shunt (10-15 nm thick, $100 \mathrm{~nm}$ wide) were evaporated at right angle of incidence. An accurate dosage of resistor wires ensured that the $\mathrm{Al}$ replicas were evaporated on the side of the resist and thus removed during lift-off. The shunt resistances $R_{s}$, made of a 3-6 $\mu \mathrm{m}$ section of $\mathrm{Cr}$, varied between 4 and $22 \mathrm{k} \Omega$; this value was deduced using the length of the shunt and the measured resistivity $r$ of the $\mathrm{Cr}$ sections in the leads. In order to compare the highly resistive $\mathrm{Cr}$ samples with those in low impedance environment, we have fabricated a reference junction consisting of thick Al leads. See Table I for description of samples with different environments.

On the dilution refrigerator, the samples were mounted inside a tight copper enclosure and the measurement leads were filtered using $0.5 \mathrm{~m}$ of Thermocoax cable.

\section{B. Effective electric circuit and the fitting formula}

The elements of the effective electric circuit in the experiment are the tunnel junction itself which is a lumped element with resistance $R_{T}$ and capacitance $C$, four leads with resistance $R$ and stray capacitance $C_{R}$ each, and shunt resistance $R_{s}$ (see Fig. 1b). An estimation shows that for voltage interval studied by us both the leads and the shunt are in the high-voltage regime where they must be considered as transmission lines: lossy lines for a shunt and leads made from $\mathrm{Cr}$ which produce the asymptotic square-root law $(1 / \sqrt{V}$-tails), while lossless lines for $\mathrm{Al}$ leads contribute to the circuit noise as pure ohmic elements (the $1 / V$-tail). Thus, both $1 / V$ - and $1 / \sqrt{V}$-tails are present simultaneously in our fitting in high voltage regime $\left(V>k_{B} T / e, e / C\right)$ which is based on the formula

$$
I=\frac{V}{R_{s}}+\frac{1}{R_{T}}\left(V-\frac{e}{2 C}+\frac{A_{1}}{V}+\frac{A_{1 / 2}}{\sqrt{V}}\right)+g V^{3} .
$$

By introducing a cubic term $g V^{3}$ into the fit we take into account the nonlinear background at large voltages. Even at large voltages $(\sim 10 \mathrm{mV})$ the strength of the cubic background does not exceed the total contribution from the power law tails. This makes it possible to resolve the power law dependence of the tail. In our measurement scheme, a small ac excitation can be added to bias current sweep to directly measure the differential conductance $d I / d V$. The fitted $g$ agrees with the parabolic background in the differential conductance measurements.

The values of parameters $A_{1}$ and $A_{1 / 2}$ expected from the theory are: 
(i) Unshunted junction with low impedance Al leads

$$
\begin{aligned}
A_{1} & =R_{K}\left(\frac{e}{2 \pi C}\right)^{2}\left(\frac{1}{R_{T}}+\sqrt{\frac{C_{R}}{L}}\right), \\
A_{1 / 2} & =0 .
\end{aligned}
$$

(ii) Unshunted junction with resistive Cr leads

$$
\begin{aligned}
A_{1} & =\frac{R_{K}}{R_{T}}\left(\frac{e}{2 \pi C}\right)^{2}, \\
A_{1 / 2} & =2 a_{1 / 2} .
\end{aligned}
$$

(iii) Shunted junction with resistive Cr leads

$$
\begin{aligned}
A_{1} & =\frac{R_{K}}{R_{T}}\left(\frac{e}{2 \pi C}\right)^{2}, \\
A_{1 / 2} & =3 a_{1 / 2} .
\end{aligned}
$$

The factors 2 and 3 in Eqs. (33) and (34) appear because four leads are equivalent to two double transmission lines shown in Fig. 1. Thus the total number of double transmission lines is 2 and 3 for the unshunted and the shunted case, respectively.7 The shunt resistance $R_{s}$ is known whereas $R_{T}$ and the capacitance $C$ as well as the parameter $A_{1}$ or $A_{1 / 2}$ are fitted to the $I V$ curve. In the case of lossless $\mathrm{Al}$ line (i) the parameter $A_{1}$ is fitted freely since it depends not only on $R_{T}$, but also on the admittance $\sqrt{C_{R} / L}$. In the case of $\mathrm{Cr}$ leads (ii,iii) $A_{1}$ is fixed by $R_{T}$ and $C$ and the parameter $A_{1 / 2}$ is fitted.

In the fabrication process the parameters (electron dose) are equal both for the shunt and the leads. This means that the capacitance and resistance per unit length are equal for $\mathrm{Cr}$ shunts and leads. The capacitance per unit length may be estimated from that of a prolate ellipsoid [14]:

$$
c_{R}=4 \pi \epsilon_{0} \epsilon_{e f f} \frac{\sqrt{1-(b / a)^{2}}}{\ln \left(a / b+\sqrt{(a / b)^{2}-1}\right)},
$$

where $a$ and $b$ are the larger and smaller radii of the ellipsoid, respectively. These are related to the length $l$, thickness $t$, and width $w$ of the Cr lead through $b \sim \sqrt{t w} \sim$ $30 \mathrm{~nm}$, and $a \sim l \sim 10 \mu \mathrm{m}$. For our silicon substrate $\epsilon_{\text {eff }} \approx 6$. Using these values we get $c_{R}=100 \mathrm{aF} / \mu \mathrm{m}$ for our typical $\mathrm{Cr}$ environment. Note that the capacitance per unit length given by Eq. (35) depends weakly on the length of the line. The fit results are compared to theoretical estimates in Table II. Using the estimated capacitance $c_{R}=100 \mathrm{aF} / \mu \mathrm{m}$ and the measured resistivity $4 \mathrm{k} \Omega / \mu \mathrm{m}$, one obtains for the attenuation coefficient $\sqrt{\omega r c_{R} / 2} \sim 0.57 \mu \mathrm{m}^{-1}$ at $1 \mathrm{mV}\left(\omega=1.6 \cdot 10^{12} 1 / \mathrm{s}\right)$.

\footnotetext{
${ }^{*}$ The shunt is considered as a two separate sections with resistance $R_{s} / 2$ each.
}

Hence, even though our Cr resistors are rather short, they can be viewed practically as infinite.

The Al leads must be characterized by the microstrip impedance

$$
\sqrt{l / c_{R}}=\frac{Z_{0}}{2 \pi \sqrt{\epsilon_{e f f}}} \ln (8 h / w) \equiv Z_{0}^{e f f},
$$

where $Z_{0}$ is the free-space impedance of $377 \Omega, h \sim 600$ $\mu \mathrm{m}$ is the distance from the ground plane, and $w \sim 200$ $\mathrm{nm}$ is the width of the strip. This yields impedance of $260 \Omega$ corresponding to capacitance per unit length of 30 $\mathrm{aF} / \mu \mathrm{m}$.

\section{Experimental results}

For presentation, it is convenient to subtract off the linear part $I=V / R_{t o t}$ with $R_{t o t}^{-1}=R_{T}^{-1}+R_{s}^{-1}$. Thus we can plot the "excess" current

$$
I_{e}=V / R_{t o t}-I+g V^{3}=\frac{1}{R_{T}}\left(\frac{e}{2 C}-\frac{A_{1}}{V}-\frac{A_{1 / 2}}{\sqrt{V}}\right)
$$

as a function of voltage $V>k_{B} T / e$.

Figure 2 shows an example of an $I_{e}$ vs. $V$-curve measured on a junction with $r=4 \mathrm{k} \Omega / \mu \mathrm{m}$ Cr leads (sample 3 ). The best fit is obtained with the lossy RC-line formula of Eq. (34) with $1 / \sqrt{V}$-tail. This yields $c_{R}=210 \mathrm{aF} / \mu \mathrm{m}$ for the specific capacitance of the Cr leads, deviating by a factor of two from the estimated value of $100 \mathrm{aF} / \mu \mathrm{m}$ of Eq. (35). The results on $1 / \sqrt{V}$-tails were found to be independent of temperature in the range $0.1-1 \mathrm{~K}$. This is in agreement with theory (see Sec. II A), according to which the tails depend only on the quantum part of Johnson-Nyquist noise.

Figure 3 shows an $I_{e}$ vs. $V$-curve of unshunted sample 2 with thick aluminum leads. As expected, the lossless transmission line formula (32) fits with impedance $\sqrt{L / C_{R}} \approx 570 \Omega$. This number agrees with Wahlgren et al. [3] who calculated $R_{e n v}=440 \Omega$ from the low-voltage data of similar unshunted single tunnel junction.

The ratio between the tails $A_{1} / V$ and $A_{1 / 2} / \sqrt{V}$ is plotted in the inset of Figure 2. Even if the magnitude $A_{1 / 2} / \sqrt{V}$ is always larger than $A_{1} / V$, their relative magnitude depends on the tunnel resistance $R_{T}$. In the strong tunneling regime $R_{T}<R_{K}, A_{1} / V$ becomes more dominant than in the samples with larger $R_{T}$. This means that one must take strong tunneling effect into account investigating the high voltage tails, and for this it is sufficient to include the ohmic tunneling resistance $R_{T}$ into the effective circuit for calculation of the JohnsonNyquist noise.

Figure 4 shows a fit using voltage-fluctuation theory Eq. (21). As well as in the other fits, a nonlinear background $g V^{3}$ was needed in the fitting. However, even the best fit yields a magnitude for the exponential 
tail which is orders of magnitude smaller than the cubic background. This is an evidence against the voltagefluctuation model.

Table II summarizes the fit results by showing the fitted $R_{T}, C$, and the parameter $\sqrt{R_{K} C_{R} / R C}$ proportional to the fitting parameter $A_{1 / 2}$. The theoretical value is obtained from calculated value of capacitance per unit length Eq.(35). The reference sample with lossless Al leads is characterized by the dimensionless impedance $R_{K} \sqrt{C_{R} / L}$ comparable to $R_{K} / Z_{0}^{e f f}$.

\section{CONCLUDING DISCUSSION}

We have studied experimentally high-voltage asymptotics of the $I V$ curves for small normal tunnel junctions and detected power-law voltage tails of $I V$ curve when approaching the linear law $V=I R+e / 2 C$. Our data are in a good agreement with theoretical predictions of the quantum theory of environment (the phase-correlation theory). Despite some numerical-factor discrepancy for high-resistance $\mathrm{Cr}$ leads which may be ascribed to inaccuracy of our effective circuit, the voltage tail grows with increasing lead admittance as predicted by the quantum theory.

Voltage tails of the form $1 / V$, typical for low-resistance leads, were experimentally studied and discussed by Wahlgren et al. within the horizon picture [3]. We have detected slower voltage tails $1 / \sqrt{V}$ using high-resistance chromium leads. We have shown that the horizon model provides a good qualitative picture also for this type of environment, but only if one takes into account that the high-resistance leads behave as lossy transmission lines in which the electromagnetic signal travels with a frequency-dependent velocity that is much less than the velocity of light.

The effect of environment is in fact a result of JohnsonNyquist noise in the electric circuit. It is important that the power-law tails at high voltages are connected only with the quantum part of Johnson-Nyquist noise. Note that in order to avoid thermal noise when studying the low-voltage part of the $I V$ curve, the condition $k_{B} T \ll e^{2} / C$ must be satisfied. For the high-voltage tails the condition may be much weaker viz. $k_{B} T \ll e V / \pi$ which is well satisfied in our experimental studies. Thus, detection of these tails in a good agreement with theory is a rather unique verification of quantum zero fluctuations in macroscopic systems.

Our experimental conditions included the case of strong tunneling when the junction resistance $R_{T}$ was less than the quantum resistance $R_{K}$. The strong-tunneling corrections to the environmental modes at high voltages can be simply incorporated by including the junction resistance $R_{T}$ into the effective electric circuit for calculation of the quantum noise.

\section{ACKNOWLEDGEMENTS}

We acknowledge interesting discussions with G.-L. Ingold and A.D. Zaikin. This work was supported by the Academy of Finland and by the Human Capital and Mobility Program ULTI of the European Union.

[1] G.-L. Ingold and Yu.V. Nazarov, in: Single Charge Tunneling, ed. H. Grabert and M.H. Devoret, (Plenum Press, N.Y., 1992), p. 48.

[2] L.J. Geerligs, V.F. Anderegg, C.A. van der Jeugd, J. Romijn, and J.E. Mooij. Europhys. Lett. 10, 79 (1989).

[3] P. Wahlgren, P. Delsing, and D.B. Haviland, Phys. Rev. B 52, R2293 (1995); P. Wahlgren, P. Delsing, T. Claeson, and D.B. Haviland, Phys. Rev. B 57, 2375 (1998).

[4] A.N. Cleland, J.M. Schmidt, and J. Clarke, Phys. Rev. B 45, 2950 (1992).

[5] A.A. Odintsov, Zh. Eksp. Teor. Fiz. 94, 312 (1988) [Sov. Phys. JETP 67, 1265 (1988)].

[6] D .S. Golubev and A.D. Zaikin, Phys. Rev. B 46, 10903 (1992).

[7] A.A. Odintsov, G. Falci, and G. Schön, Phys. Rev. Lett. B 44, 13089 (1991).

[8] P. Joyez and D. Esteve, Phys. Rev. B 56, 1848 (1997).

[9] Sh. Farhangfar, J.J. Toppari, Yu. Pashkin, A.J. Manninen, and J.P. Pekola, Europhys. Lett. 43, 59 (1998).

[10] G. Schön and A.D. Zaikin, Phys. Rep. 198, 237 (1990).

[11] Yu.V. Nazarov, Zh. Eksp. Teor. Fiz. 95, 975 (1989) [Sov. Phys. JETP 68, 561 (1989)].

[12] J.P. Kauppinen and J.P. Pekola, Phys. Rev. Lett., 77 3889, (1996).

[13] M. Büttiker and R. Landauer, IBM J. Res. Dev. 30, 451 (1986).

[14] L.D. Landau, E.M. Lifshitz and L.P. Pitaevskii, Electrodynamics of Continuous Media (Pergamon, Oxford, 1984).

TABLE I. Measured shunted junctions. The value of $R_{s}$ is estimated from the known wire resistivity $r$.

\begin{tabular}{c|c|c}
\hline \hline sample & $R_{s}(k \Omega)$ & Description of environment \\
\hline 1 & $\infty$ & Cr leads only, $r=4 \mathrm{k} \Omega / \mu m$ \\
\hline 2 & $\infty$ & Al leads only \\
\hline 3 & 22.4 & Cr shunt /leads, $r=4 \mathrm{k} \Omega / \mu m$ \\
\hline 4 & 4.2 & Cr shunt $/$ leads, $r=1.5 \mathrm{k} \Omega / \mu m$ \\
\hline \hline
\end{tabular}


TABLE II. Fit results. $R_{T}$ and $C$ are fitted to the data. Parameters $\sqrt{R_{K} C_{R} / R C}$ and $R_{K} \sqrt{C_{R} / L}$ are calculated from the fitted parameters $A_{1 / 2}$ and $A_{1}$, respectively. These are compared with theoretical estimates (see text).

\begin{tabular}{c|c|c|c|c}
\hline \hline \multirow{2}{*}{ sample } & \multirow{2}{*}{$R_{T}(\mathrm{k} \Omega)$} & \multirow{2}{*}{$C(\mathrm{fF})$} & \multicolumn{3}{|c}{$\sqrt{R_{K} C_{R} / R C}$} \\
& & & fit & theory \\
\hline 1 & 4.3 & 1.1 & 1.11 & 0.77 \\
\hline 3 & 11.1 & 1.0 & 1.16 & 0.80 \\
\hline 4 & 18.4 & 0.1 & 3.84 & 4.15 \\
\hline \hline sample & $R_{T}(\mathrm{k} \Omega)$ & $C(\mathrm{fF})$ & \multicolumn{2}{|c}{$R_{K} \sqrt{C_{R} / L}$} \\
& & & fit & $R_{K} / Z_{0}^{\text {eff }}$ \\
\hline 2 & 76.0 & 0.7 & 45.3 & 99 \\
\hline \hline
\end{tabular}

a)

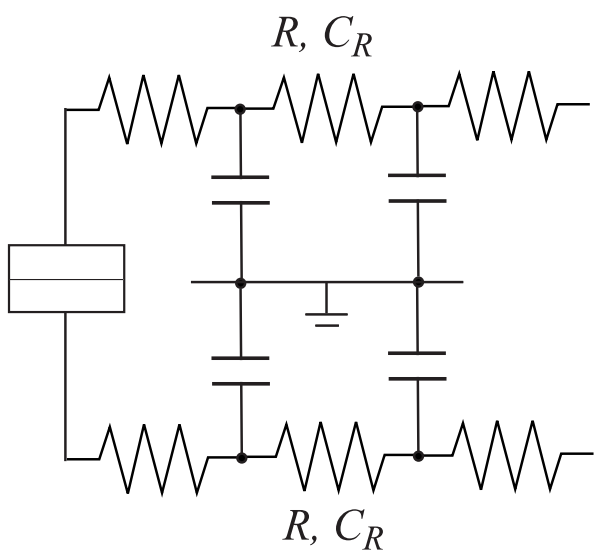

b)

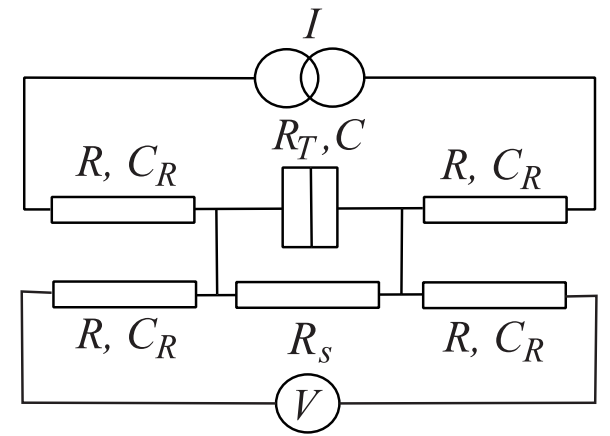

FIG. 1. a) High frequency model for resistive measurement leads. Microstrip leads are viewed as transmission lines with the total capacitance $C_{R}$ and resistance $R$ per each. b) Measurement setup.

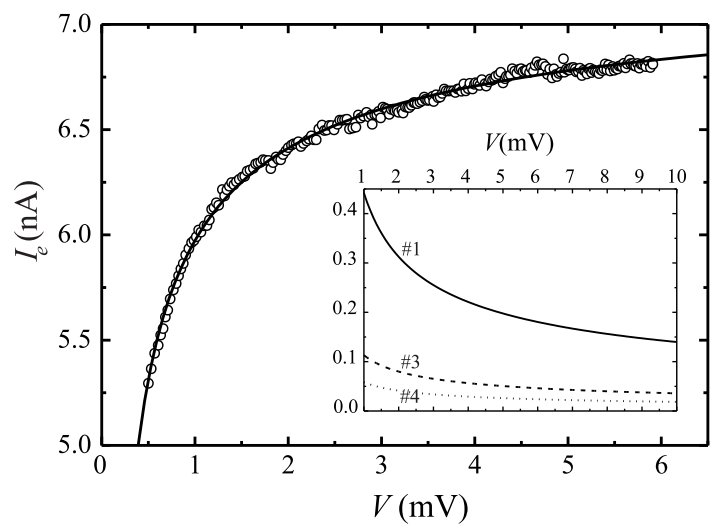

FIG. 2. Reduced "excess" current $I_{e}$ vs. voltage $V$ for sample 3 with tunneling resistance $R_{T}=11.1 \mathrm{k} \Omega$ and a resistive $0.1 \times 10 \mu \mathrm{m}$ Cr shunt $\left(R_{s}=22.4 \mathrm{k} \Omega\right)$. Solid line illustrates fit using lossy RC transmission line formulas Eq. 34. Inset shows the ratio between two tail contributions arising from tunnel junction and transmission line. 


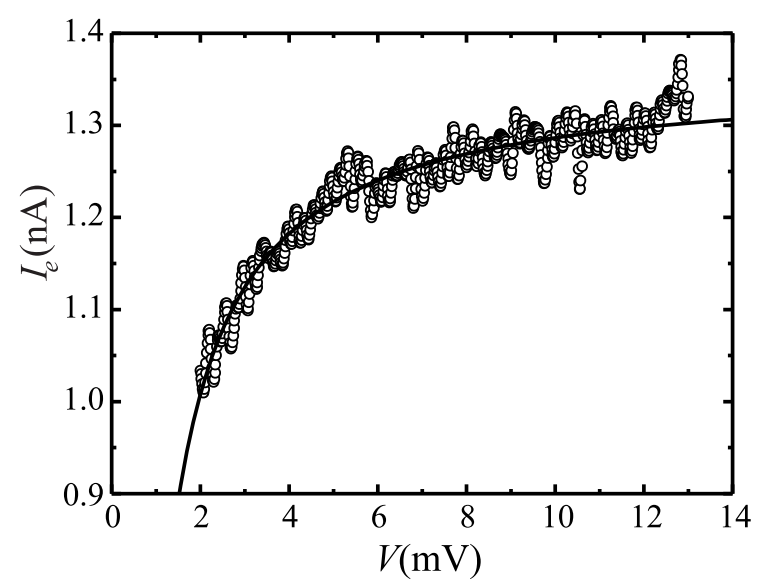

FIG. 3. "Excess" current vs. voltage for an unshunted full Al sample with tunneling resistance $R_{T}=76.0 \mathrm{k} \Omega$. Fit using lossless transmission line formulas Eq. (32).

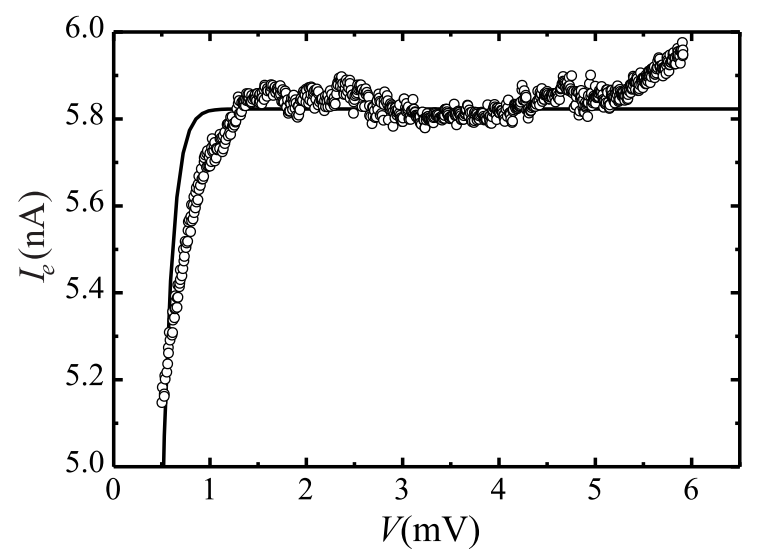

FIG. 4. Fit to sample 3 with $R_{s}=22.4 \mathrm{k} \Omega$ using voltagefluctuation theory. The background cubic nonlinearity used in the fitting formula was orders of magnitude larger than the exponential tail of Eq. (21). 